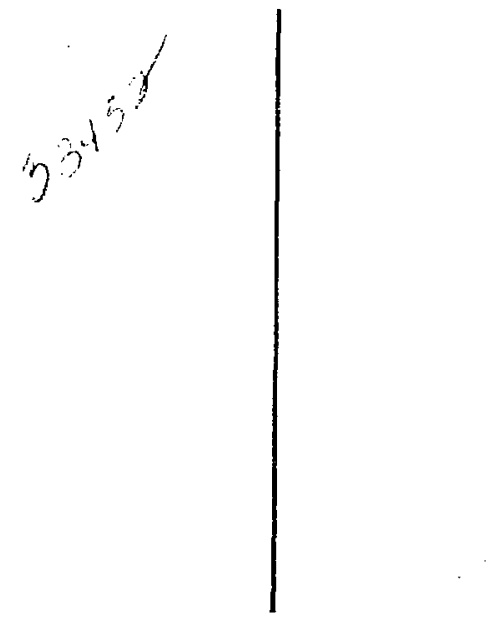

Nonlinear Diffusion of Pulsed Magnetic Fields in Planar and Cylindrical Plasmas 
This report was prepared as an account of work sponsored by the United States Government. Neither the United States nor the United States Atomic Energy Commission, nor any of their employees, nor any of their contractors, subrontractors, or their emproyees, makes any warranty, express or implied, or assumes any legal liability or responsibility for the accuracy, completeness or usefulness of any information, apparatus, product or process disclosed, or represents that its use would not infringe privately owned rights.

Printed in the United States of America. Available from National Technical Information Service

U. S. Department of Commerce 5285 Port Royal Road

Springfield, Virginia 22151

Price: Printed Copy $\$ 200$; Microfiche $\$ 0.95$

ef. 00 


\section{Nonlinear Diffusion of Pulsed Magnetic Fields in Planar and Cylindrical Plasmas}

by

Albert Haberstich

This report was prepared as an account of work sponsored by the United States Government. Neither the United States nor the United States Atomic Energy Commission, nor any of their employees, nor any of their contractors, subcontractors, or their employees, cir cons, mes pegal liability or responsibility for the accuracy, completeness or usefulness of any information, apparatus, would not infringe privately owned rights. 
NONLINEAR DIFFUSION OF PULSED MAGNETIC FIELDS

IN PLANAR AND CYLINDRICAL PLASMAS

by

Albert Haberstich

\begin{abstract}
The one-dimensional, one-component, nonlinear diffusion of pulsed magnetic fields in planar and cylindrical geometries is examined. The diffusion coefficient $\mu$ depends abruptly on the current density $j$ associated with the gradient of the magnetic field. The coefficient $\mu$ is large when $j \geq j_{1}$, small when $j \leq j_{2}$, and depends linearly on $j$ in the interval $j_{2}<j<j_{1}$, where $j_{1}$ and $\vec{j}_{2}$ are given current densities. A traveling wave solution is obtained in planar geometry and it is found that the product $\mu j$ remains continuous in space in the $l$ imit $\left(j_{1}-j_{2}\right) \rightarrow 0$. Analytic and finite difference solutions of the traveling wave problem are compared, numerical stability conditions are found, and an estimate of the numerical error is obtained. The pulsed magnetic field problem is then solved numerically in the two geametries. Large portions of the magnetic field profiles exhibit a nearly uniform current density distribution at some time during the diffusion.
\end{abstract}

\section{INTRODUCTION}

Plasmas are compressed and heated in theta and $Z$ pinches by a fast rising external magnetic field. This magnetic field is produced by an external coil in the theta pinch and by a current running through the discharge in the 2 pinch.

Due to the finite electrical resistivity of the pinch, the pulsed magnetic field gradually diffuses into the plasma. The rate of diffusion was measured in both configurations and was found to be more rapid than expected from classical electrical resistivity. ${ }^{1-4}$ The diffusion is enhanced during the early stage of the pinch when the current density induced by the magnetic field gradient is concentrated in a narrow discharge region. The anomalously large resistivity appears to be due to a microinstability that is excited when the absolute value $j$ of the current density exceeds a certain threshold $j_{c}{ }^{1-3}$

This effect can be included in hydromagnetic calculations by allowing the electrical resistivity to become anomalous whenever the local value of $j$ exceeds $j_{c} \cdot{ }^{1-5}$ The hydromagnetic calculation then involves solving a highly nonlinear diffusion problem. We propose to explore some of the analytical and numerical properties of this diffusion problem. We write the diffusion equation in a onecomponent, one-dimensional form and assume that the diffusion coefficient $\mu$, associated with the electrical resistivity of the plasma, is of the form shown in Fig. 1. The problem then becomes similar to a Stefan problem. ${ }^{6}$ The diffusion coefficient of the Stefan problem, however, would depend on $B$ rather than on the spatial derivative of $B$. Therefore, the stefan solution does not seem to be directly applicable.

Here we seek a traveling wave solution of the diffusion equation in planar geometry. We divide this solution into the three regions, where $:>j_{1}$, $\mathrm{j}<\mathrm{j}_{2}$, and $j_{2} \leq \mathrm{j} \leq \mathrm{j}_{1}$, and find that the spatial width of the transition region $j_{2} \leq j \leq j_{1}$ remains finite in the limit $\left(j_{1}-j_{2}\right) \rightarrow 0$. The product $\mu j$, which is proportional to the electric field in the plasma, then remains continuous in space. 


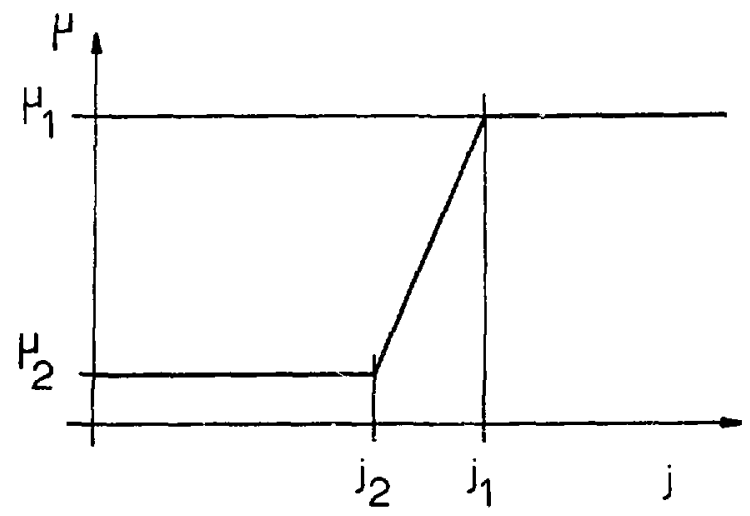

Fig. 1. Diffusion coeficicient $\mu$ as a function of the absolute value of the current density $j$.

We also solve the problem numerically using finite difference explicit and implicit schemes. We write these schemes in such a way as to conserve magnetic fluxes. We find that, for sirall time and space increments, the numerical solutions can be stable and that they agree closely with the analytic traveling wave solution. It appears, therefore, that the scheme used in our earlier numerical MHD calculations ${ }^{4}$ is adequate to hand le this type of diffusion problem, at least in a planar approximation.

We make use of the explicit difference scheme to solve basic pulsed magnetic field diffusion problems in planar and cylindrical geometries, and find that large portions of the pinch may become part of the transition region, and may therefore exhibit a uniform current density distribution.

\section{ANALYTIC TRAVELING WAVE SOLUTION IN PLANAR GEOMETRY}

The nonlinear diffusion problem discussed above can be reduced to the one-dimensional, onecomponent diffusion equation

$$
\frac{\partial B}{\partial t}=\frac{\partial}{\partial x}\left(\mu \frac{\partial B}{\partial x}\right),
$$

where $t$ is the time, $x$ is the $x$-coordinate, $B$ is the magnetic field, and $\mu$ is the diffusion coefficient of the form shown in Fig. 1 .

In Fig. 1, $j=|\partial B / \partial x|$ is the absolute value of the current densizy. The coefficients $\mu_{1}$ and $H_{2}$ are conetants, respectively proportional to the anomalous and classical resistivities of the plasma.
The transition from ${ }_{1} 1$ to : $_{2}$ takes place linearly over the current density interval $j_{1}$ to $j_{2}$. Thus,

$$
\mu= \begin{cases}\mu_{2} & , j<j_{2}, \\ \mu_{c}+\frac{\mu \mu}{\Delta j}\left(j-j_{c}\right), & j_{2} \leq j \leq j_{1}, \\ \mu_{1}, & j>j_{1},\end{cases}
$$

where

$$
\begin{aligned}
& j_{c}=\frac{1}{\varepsilon}\left(j_{1}+j_{2}\right), \quad \Delta j=j_{1}-j_{2}, \\
& \mu_{c}=\frac{i}{E}\left(\mu_{1}+\mu_{2}\right), \text { and } \Delta \mu=\mu_{1}-\mu_{2} .
\end{aligned}
$$

We assume that $\mu_{1}$ is always larger or equal to $\mathrm{H}_{2}$. Equations (1) and (2) have an analytic traveling wave solution, which is defined as a solution that travels undistorted at constant velocity $v$, here chosen in the positive x-direction. 7 In Fig. $2, B(x, t)$ then consists of a leading edge $x>x_{2}$ where $j<j_{2}$, a transition region $x_{1} \leq x \leq x_{2}$ where $j_{2} \leq j \leq j_{1}$, and a region $x<x_{1}$ where $j>j_{1}$. The boundaries $x_{1}$ and $x_{2}$ satisfy $x_{1}=x_{10}+v t$ and $x_{2}=x_{20}+v t$. Either $x_{10}$ or $x_{20}$ can be chosen arbitrarily.

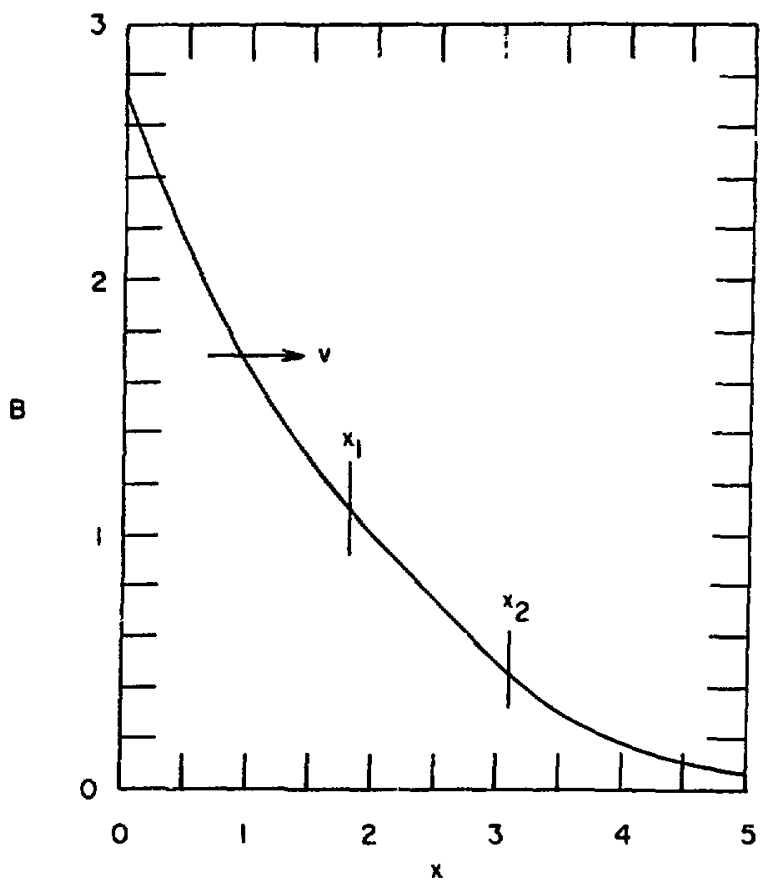

Fig. 2. Analytic traveling wave solution for $v=1$, $\mu_{1}=2, \mu_{2}=1, j_{c}=0.5$, and $\Delta j=0.1$. 
We solve Eqs. (1) and (2) in the three regions, match boundary conditions at $x_{1}$ and $x_{2}$, and obtain

$$
\begin{aligned}
& B=\frac{\alpha_{2} j_{2}}{v} \exp \left(-v \frac{x-x_{2}}{\alpha_{2}}\right) \\
& x>x_{2}, \\
& B=\frac{{ }^{\prime} 1}{v} \exp \left(-v \frac{x-x_{1}}{\iota_{1}}\right) \\
& x-x_{1} \text {, }
\end{aligned}
$$

and

$$
\begin{aligned}
& x-x_{1}= \frac{1}{v}\left[\left(2 a_{1}+b\right)-\left(b^{2}+4 a v B\right)^{\frac{1}{2}}\right. \\
&\left.+b \ln \frac{b+\left(b^{2}+4 a v B\right)^{\frac{1}{2}}}{2\left(\mu_{1}+b\right)}\right], \\
& x_{1} \leq x \leq x_{2},
\end{aligned}
$$

where

$$
a=\frac{\Delta t}{\Delta j} \quad \text { and } \quad b=\frac{\mu_{1} j_{2}-\mu_{2} j_{1}}{\Delta j} \text {. }
$$

The ratio of the magnetic fields $B\left(x_{1}\right)=B_{1}$ and $B\left(x_{2}\right)=B_{2}$ at the boundaries of the transition region follows from Eqs. (3) and (4),

$$
\frac{B_{1}}{B_{2}}=\frac{\mu_{1} j_{1}}{\mu_{2} j_{2}} \text {. }
$$

Taking the spatial derivative of Eqs. (3) through (5), we obtain the absolute current densities

$$
\begin{aligned}
& j=j_{2} \exp \left(-v \frac{x-x_{2}}{\mu_{2}}\right), \\
& j=j_{1} \exp \left(-v \frac{x-x_{1}}{\mu_{1}}\right), \\
& x<x_{1},
\end{aligned}
$$

and

$$
\begin{array}{r}
x-x_{1}=\frac{1}{v}\left[2 a\left(j_{1}-j\right)+b \ln \frac{j}{j_{1}}\right], \\
x_{1} \leq x \leq x_{2} .
\end{array}
$$

The width $\left(x_{2}-x_{1}\right)$ of the transition region is a special case of Eq. (8),

$$
x_{2}-x_{1}=\frac{1}{v}\left(2 i_{L}+b \ln \frac{j_{2}}{j_{1}}\right) \text {. }
$$

It is of interest to note that ${ }^{8}$

$$
\lim _{\Delta \mathrm{j} \rightarrow 0}\left(x_{2}-x_{1}\right)=\frac{\Delta}{v} \text {. }
$$

Thus, the transition region width remains finite in the limit $\Delta \mathrm{j} \rightarrow 0$. Letting $\Delta \mathrm{j} \rightarrow 0$ in Eq. (8), we find that $j$ becomes a linear function of $x$ in the transition region and that the product $\mu j$, which is proportional to the electric field in the plasma, remains continuous throughout $x$-space.

\section{NUMERICAL TRAVELING WAVE SOLUTION IN PLANAR GEOMETRY}

Equations (1) and (2) are solved with the following magnetic flux conserving explicit and implicit difference schemes ${ }^{9}$

$$
\begin{aligned}
B_{i+\frac{i}{2}}^{n+1}-B_{i+\frac{i}{2}}^{n}= & \frac{\Delta t}{(\Delta x)^{2}}\left[\left(B_{i+3 / 2}^{n}-B_{i+\frac{i}{i}}^{n}\right) i_{i+1}^{n}\right. \\
& \left.-\left(B_{i+\frac{i}{2}}^{n}-B_{i-\frac{i}{2}}^{n}\right) \mu_{i}^{n}\right]
\end{aligned}
$$

and

$$
\begin{aligned}
B_{i+\frac{1}{\varepsilon}}^{n+1}-B_{i+\frac{i}{2}}^{n}= & \frac{\Delta t}{(\Delta x)^{2}}\left[\left(B_{i+3 / 2}^{n+1}-B_{i+\frac{1}{2}}^{n+1}\right) \mu_{i+1}^{n+1}\right. \\
& \left.-\left(B_{i+\frac{1}{2}}^{n+1}-B_{i-\frac{1}{2}}^{n+1}\right) \mu_{i}^{n+1}\right],
\end{aligned}
$$

respectively, where $t=n \Delta t$ and $x=i \Delta x$. The diffusion coefficients $\mu_{i}^{n}$ and $u_{i}^{n+1}$ are siven by

$$
\mu_{i}^{n, n+1}=\left\{\begin{array}{ll}
\mu_{2} & , j_{i}^{n, n+1}<j_{2} \\
u_{c}+\frac{\Delta u}{\Delta j}\left(j_{i}^{n, n+1}-j_{c}\right), & j_{2} \leq j_{i}^{n, n+1} \leq j_{1}, \\
\mu_{1} & j_{i}^{n, n+1}>j_{1}
\end{array},\right.
$$

and

$$
j_{i}^{n, n+1}=\frac{1}{\Delta x}\left(B_{i-\frac{1}{2}}^{n, n+1}-b_{i+\frac{1}{2}}^{n, n+1}\right) .
$$


In the regions of uniform ,1, Eq. (11) is stbject to the numerical stability condition

$$
\frac{\Delta t}{(\Delta x)^{2}}+\leq 1 / 2
$$

whereas $\mathrm{Eq}$. (12) is always stable. ${ }^{9}$ The numerical stability of the explicit scheme in the transition region is derived in Appendix $A$ and is given sy

$$
\frac{\Delta t}{(\Delta x)^{2}} \mu_{c} \leq \frac{1}{2} \frac{1}{1+\frac{\Delta \mu}{\mu_{c}}\left(1+\frac{j c}{\Delta j}\right)} \text {. }
$$

The advanced diffusion coefficient in the transition region is not known a priori. We therefore determine $\mu_{i}^{n+1}$ by the iterative procedure

$$
\mu_{i}^{n+1, m+1}=(1-A) \mu_{i}^{n+1, m}+A \bar{\mu}_{i}^{n+1, m} \text {, }
$$

where $0<A \leq 1$ is a relaxation factor. The diffusion coeficicient $u_{i}^{n+l}, m^{+1}$ is used in the new iteration, $\mu_{i}+1, m$ is the coefficient used during the mth iteration, and $\bar{\mu}_{i}{ }^{n+1, m}$ is calculated by Eqs. (13) and (14), using the magnetic fields obtained by the mth iteration. This procedure is repeated until $\bar{\mu}_{i}{ }^{n+1, m+1}$ approaches $\mu_{i}^{n+1, m+1}$ to within a certain accuracy. The stability of the implicit scheme then depends on the convergence of che iteratior scheme. The stability condition, derived in Appendix $B$, is

$$
\frac{\Delta t}{(\Delta x)^{2}} \mu_{c} \leq \frac{\frac{2}{4}-1}{\frac{\Delta_{u}}{\mu_{c}}\left[\left(\frac{1}{A}-1\right)+\frac{j_{c}}{\Delta j}\right]-\left(\frac{2}{A}-1\right)}
$$

The accuracy of the two numerical schemes can be determined by comparing numerica.1 solutions of the traveling wave problem with the analytic solution. The numerical error in the uniform $\mu$ regions can be predicted. We define the relative error $\epsilon$ as

$$
\varepsilon=\left(\mathrm{B}_{\text {numerical }}-\mathrm{B}_{\text {analytic }}\right) / \mathrm{B}_{\text {analytic }},
$$

and find it to the lowest order in $\Delta x$ and $\Delta t$,

$$
\epsilon=\left[\frac{1}{12} \frac{v^{4}}{u^{3}}(\Delta x)^{2} \mp \frac{1}{2} \frac{v^{4}}{\mu^{2}} \Delta t\right] t \text {, }
$$

where the uppe: and lower signs apply to the explicit and implicit schemes, respectively. This numerical error can be eliminated by adding a small correction $5 L$ to the diffusion coefficient. For small values of $A x$ and $\Delta t$ we find that

$$
5 \alpha=\frac{\mu-v-\frac{1}{6}}{\left(\frac{\mu}{v \Delta x}\right)^{2}+v+\frac{1}{12}},
$$

for the implicit scheme, and that

$$
S_{t: 1}=\frac{\nu}{2} \frac{v-\frac{1}{6}}{\left(\frac{i L}{v \Delta x}\right)^{2}+\frac{i}{12}}
$$

for the explicit scheme, where $v=u \Delta t /(\Delta x)^{2}$.

The accuracy of the numerical schemes in the transition region is determined experimentally. Starting at $t=0$, with the initial state prescribed by Eqs. (3) through (5), we numerically advance the traveling wave solution shown earlier in Fig. 2. The values of $B$ at the boundaries $x=0$ and $x=5$ are varied at the rates predicied by Eqs. (3) and (4). Here we add the small correction $\delta \mu$ to the diffusion coefficient to allow for the error anticipated in the regions of constant $\mu$, and thus delay the effect of the bolindaries $x=0$ and $x=5$ on the numerical error in the transition region.

A typical observed error $\varepsilon$ is shown in Fig. 3 as a function of $x$ at four consecutive times in the diffusion. The shape of $\varepsilon(x)$ varies as the transition region traverses the mesh structure. A plot of the peak absolute error $|\hat{\varepsilon}|$ as a function of time is shown in Fig. 4, whereas $\langle|\epsilon|\rangle$, the absolute value of $\varepsilon$ averaged over the interval $0 \leq x \leq 5$ as a function of time, is shown in Fig.

5. Figures 4 and 5 were obtained with the implicit scheme with $\Delta \mathbf{x}=0.033$. Similar calculations have been performed with $\Delta x=0.05$ and the two calculations have been repeated with the explicit scheme.

The peak value of $|\widehat{\varepsilon}|$ at time nearest $t=0.1$ is plotted in Fig. 6 as a function of $\Delta t$ for the implicit scheme for the two values of $\Delta x$. The peak error is essentially independent of $\Delta t$. For small values of $\Delta t,|\hat{\varepsilon}|$ is proportional to $(\Delta *)^{2}$. Mean values of $\langle|\varepsilon|\rangle$ at time $t=0.1$ are plotted in Figs. 7 and 8 as finctions of $\Delta t$ for $\Delta x=0.05$. Figure 7 

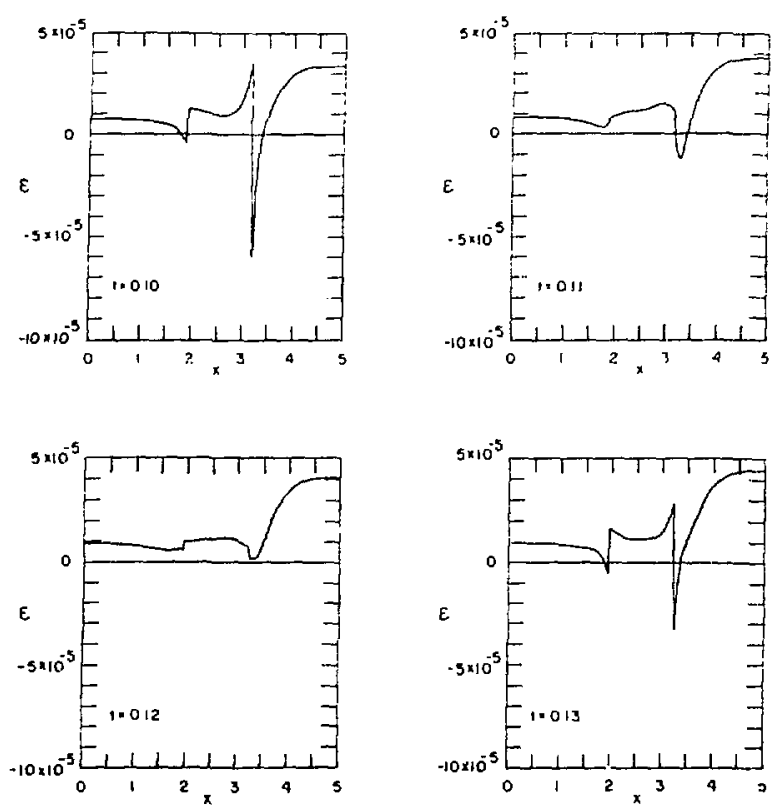

Fig. 3. Observed numerical error $\epsilon$ at times $t=0.1,0.11,0.12$, and 0.13 of the traveling wave solution, Fig. 2, for $\Delta x=0.033$ and $\Delta t=5 \times 10^{-4}$. Inplicit scheme.

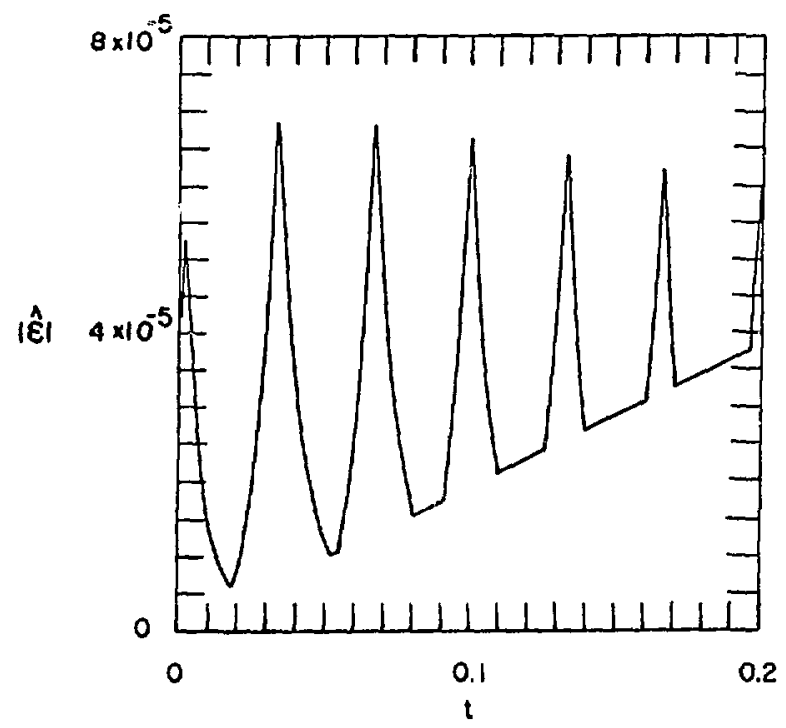

Fig. 4. Peak error $|\hat{\varepsilon}|$ as a function of time for the conditions of Fig. 2 for $\Delta x=0.033$ and $\Delta t=2 \times 10^{-4}$. Implicit scheme.

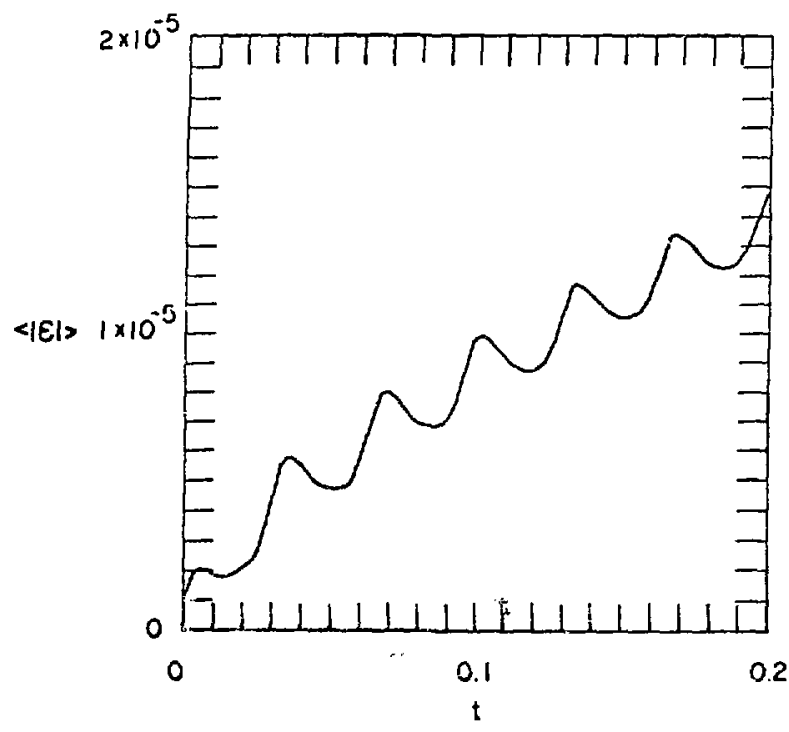

Fig. 5. Mean error $\langle|\epsilon|\rangle$ over the interval $0 \leq x \leq 5$ as a function of time for the conditions of Fig. 2 for $\Delta x=0.033$ and $\Delta t=2 \times 10^{-4}$. Implicit scheme.

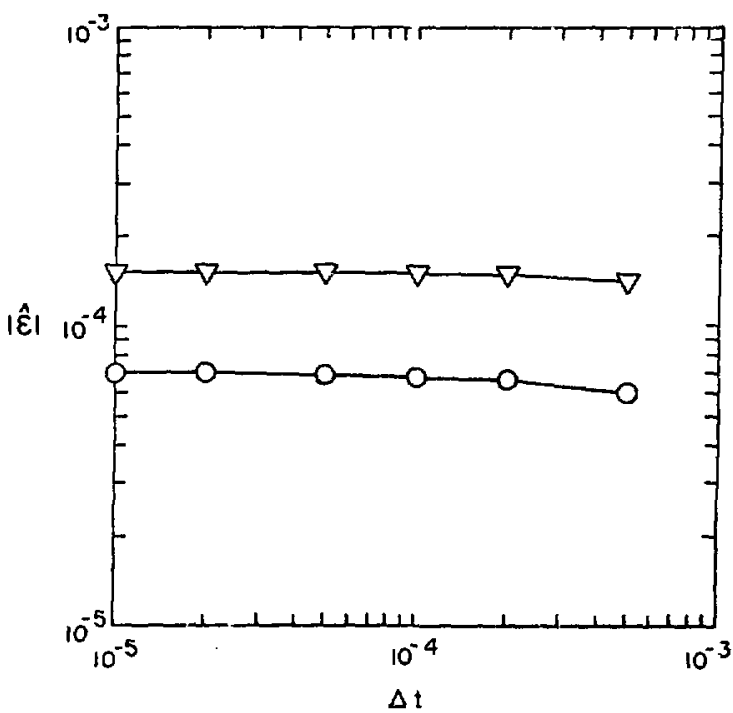

Fig. 6. Feak value of $|\hat{\epsilon}|$ at time nearest $t=0.1$ as a function of $\Delta t$ for the conditions of Fig. 2 with $\Delta x=0.05$ (triangles) and $\Delta \mathrm{x}=0.033$ (circles). Impicit scheme. 


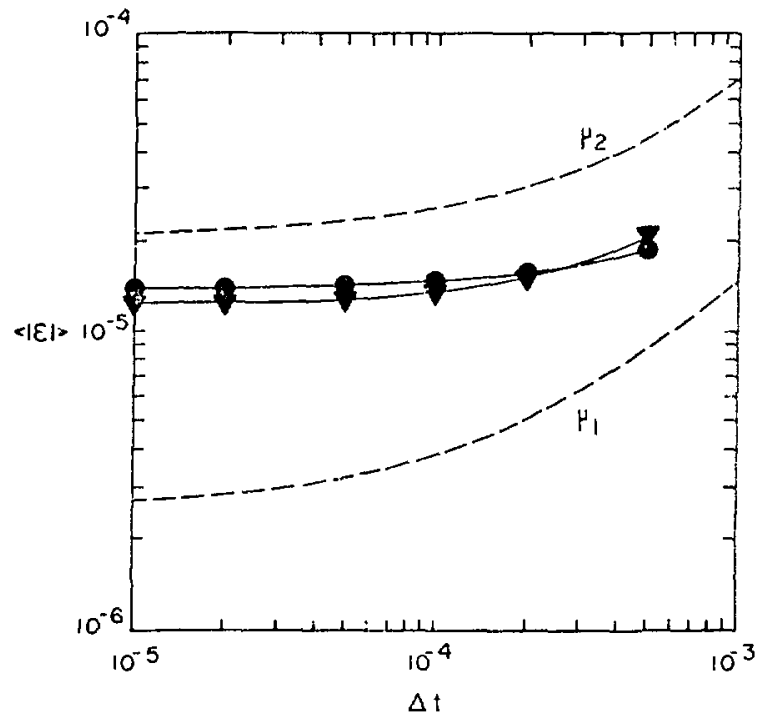

Fig. 7. Mean error $\langle|\epsilon|\rangle$ at time $t=0.1$ as a function of $\Delta t$ for the conditions of Fig. 2 and $\Delta x=0.05$. | | $\mid$ averaged over the interval $0 \leq x \leq 5$ (triangles) and over the transition $\bar{r}$ egion only (circles). Implicit scheme.

corresponds to the implicit scheme and Fi.g. 8 to the explicit solution. The triangles indicate averages over the interval $0 \leq x \leq 5$, whereas the circles correspond to averages over the transition region only. The dashed lines obtained from Eq. (19) show the error associated with the regions of uniform $\mu_{1}$ and $\mu_{2}$. The $\Delta x=0.033$ calculation indicates that for small values of $\Delta t,\langle|\epsilon|\rangle$ is proportional to $(\Delta x)^{2}$ as in the uniform $\mu$ case. For large $\Delta t,\langle|\epsilon|>$ increases with $\Delta t$ in the implicit case. The large at region is not accessible to the explicit scheme.

The results obtained in this section indicate that the explicit and implicit schemes can be stable and that the numerical errors can be made neglizibly small by choosing a small $\Delta x$, which in turn calls for a small value of $\Delta t$ to satisfy stability requirements.

\section{DIFFUSION OF PULSED MAGNETIC FIELD IN PLANAR GEOMETRY}

Having established the stability and accuracy of Eqs. (11) and (12), we san use these schemes to calculate the diffusion of pulsed magnetic fields into a semi-infinite plasma. The plasma is uniform and extends over the region $x=0$ to infinity. The

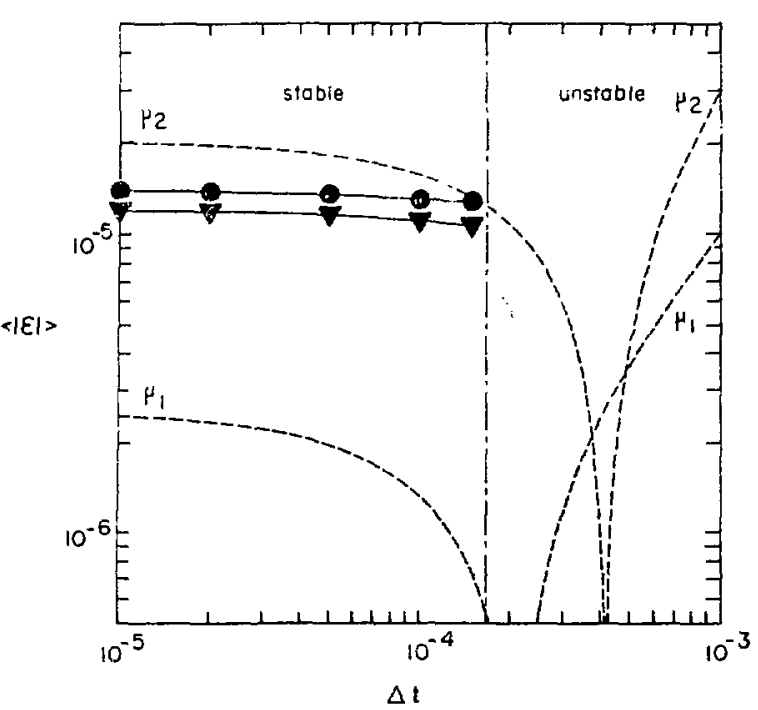

Fig. 8. Same as Fig. 7, but explicit scheme.

magnetic field is uniform in the region $x=0$ to minus infinity and is pulsed from zero to $B=B_{0}$ at time $t=0$. Thus, in addition to Eqs. (1) and (2), we must satisfy the initial and boundary conditions.

$$
B=\left\{\begin{array}{lll}
0, & x>0, & t<0, \\
B_{0}, & x=0, & t \geq 0 .
\end{array}\right.
$$

Figure 9 shows an explicit solution obtained with $j_{c}=0.5, \Delta j=0.01, \mu_{1}=2$, and $\mu_{2}=0.02$. The magnetic field satisfies Eq. (22) at $x=0$, whereas $B=0$ at $x=5$. A transttion region is seen to develop. Due to the small value of $\Delta j$, $B(x)$ is nearly linear in the transition region. The speed at which the transition region propagates can be predicted as a function of its width. In the limit $\mu_{2} \rightarrow 0$

$$
\frac{\mathrm{dx}_{2}}{\mathrm{dt}}=\frac{\mu_{1}}{\mathrm{x}_{2}-\mathrm{x}_{1}}
$$

For a semi-infinite plasma, this expression remains valid as long as the transition region does not extend to the origin $\mathrm{x}=0$.

\section{DIFEUSION OF PULSED MAGNETIC FIELDS IN CYLINDRICAL GEOMETRY}

The plasma here is cylindrical, axially and azimuthally symmetric, and has the radius $r_{a}$. The magnetic field can have two components, $B_{z}$ and $B_{\theta^{*}}$ We consider the two cases separately. 


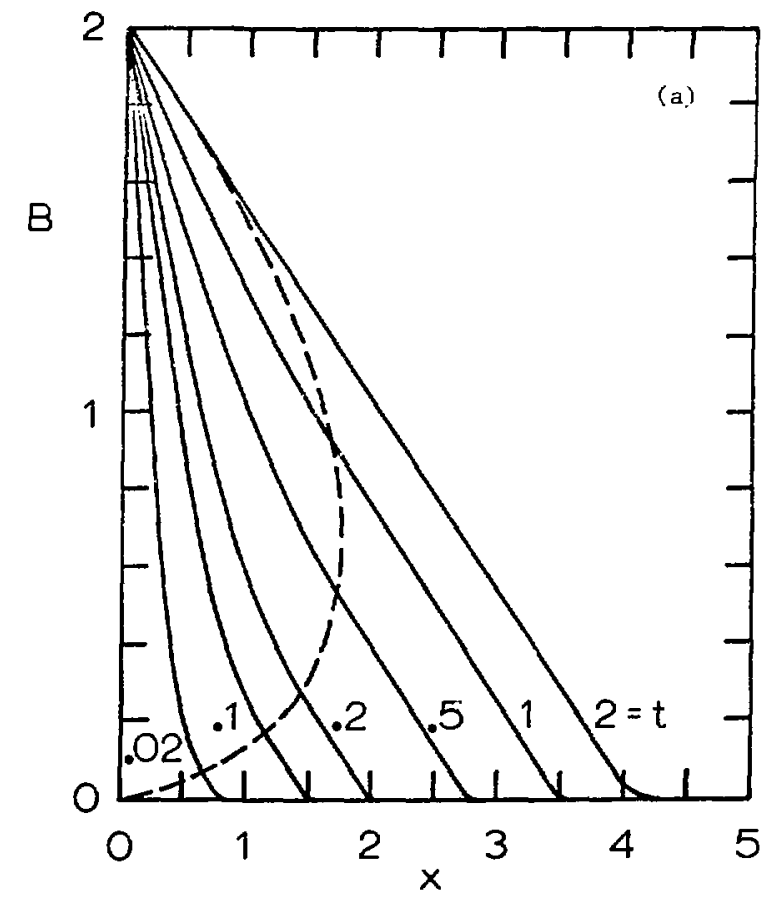

(a) $B(x)$ as a function of time. The dashed line shows the trajectory of $\mathrm{B}\left(\mathrm{x}_{1}\right) ; \mathrm{B}\left(\mathrm{x}_{2}\right)$ is close to the abscissa.

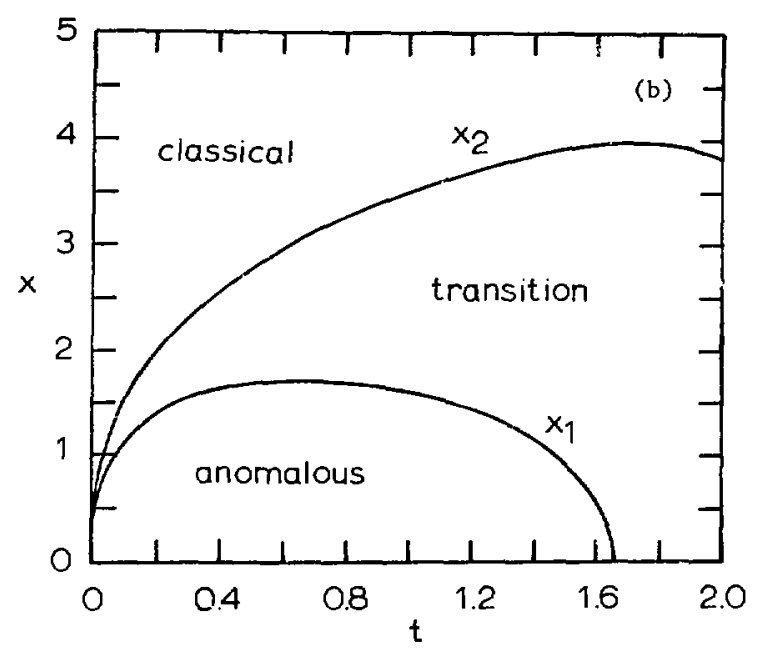

(b) $x_{1}$ and $x_{2}$ as functions of time.

Fig. 9. Diffusion of pulsed magnetic field in planar geometry. $B_{0}=2, \mu_{1}=2$, $\mu_{2}=0.02, j_{c}=0.5$, and $\Delta j=0.01$.
The $\mathrm{B}_{z}$ field case is typical of a theta pinch. $B_{z}$ is uniform in the outer region $r>r_{a}$ and is pulsed from zeru to $B_{z}=B_{z o}$ at time $t=0$. The initial and boundary conditions are

$$
B_{z}= \begin{cases}0, & r<r_{a}, t \leq 0, \\ B_{z 0}, & r=r_{a}, t \geq 0 .\end{cases}
$$

The diffusion equation in cylindrical coordinates becomes

$$
\frac{\partial \mathrm{B}_{z}}{\partial \mathrm{t}}=\frac{1}{\mathrm{r}} \frac{\partial}{\partial \mathrm{r}}\left(\mu \mathrm{r} \frac{\partial \mathrm{B}_{z}}{\partial \mathrm{r}}\right) \text {, }
$$

where $\mu$ is now defined in terms of $j=\left|j_{\theta}\right|$, with

$$
j_{\theta}=-\frac{\partial \mathrm{B} z}{\partial \mathrm{r}},
$$

and $j_{\theta}=0$ on axis.

Equation (25) can be solved by finite difference schemes similar to Eqs. (11) and (12). A1though the accuracy of these schemes has not been checked in cylindrical geometry, it is believed to be comparable to that of the linear schemes. It might be necessary, however, to study this point fur ther.

The explicit difference scheme in cylindrical coordinates can be written as

$$
\begin{aligned}
B_{z, i+\frac{1}{2}}^{n+1}-B_{z, i+\frac{1}{2}}^{n} & = \\
& \frac{\Delta t}{(\Delta r)^{2}} \frac{1}{r_{i+\frac{1}{2}}}\left[r_{i+1} \mu_{i+1}^{n}\left(e_{z, i+3 / 2}^{n}-B_{z, i+\frac{1}{2}}^{n}\right)\right. \\
& -r_{i} \mu_{i}^{n}\left(B_{z, i+\frac{1}{2}}^{n}-B_{z, i}^{n} \cdot\right],
\end{aligned}
$$

where the $\mathrm{B}_{z}$ flux is conserved. The diffusion coefficient $\mu_{i}^{n}$ is defined in terms of Eq. (13) with

$$
j_{i}^{n}=\frac{1}{\Delta r}\left(B_{z, i+\frac{1}{2}}^{n}-B_{z, i-\frac{1}{2}}^{n}\right) \text {. }
$$

Figures 10 and 11 show the numerical results obtained with $j_{c}=0.5, \Delta j=0.01, \mu_{1}=2$, and $\mu_{2}=0.02$. $r_{1}$ and $r_{2}$ are the outer and inner boundaries of the transition region, respectively. We call the case shown in Fig. 10 supercritical, meaning that $\mathrm{B}_{z 0} \geq \mathbf{r}_{a} \mathrm{j}_{\mathrm{c}}$. The transition region here 


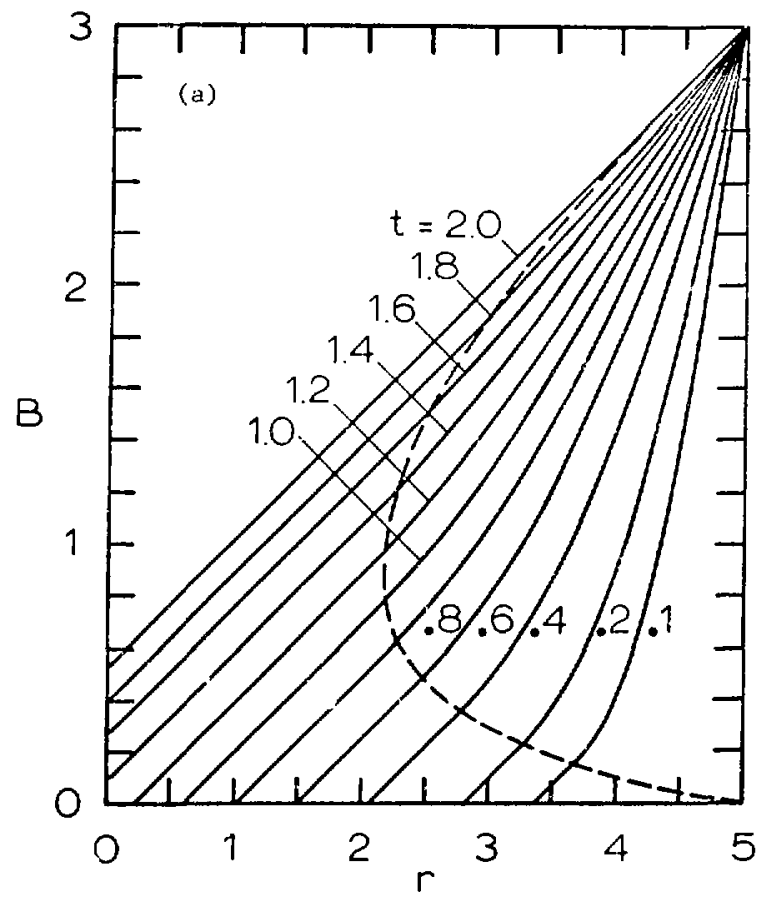

(a) $B_{z}(r)$ as a function of time. The dashed line rapresents $B_{z}\left(r_{1}\right) ; B_{z}\left(r_{2}\right)$ is close to the abscissa.

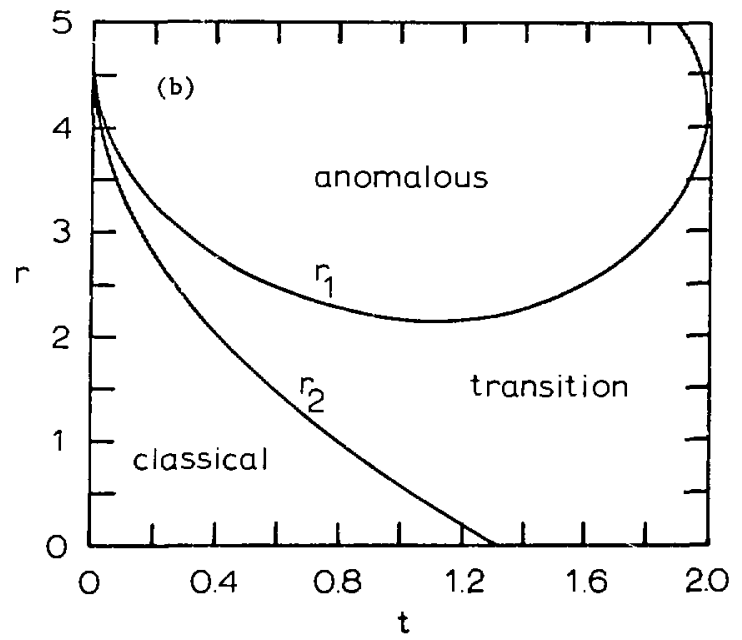

(b) $r_{1}$ and $r_{2}$ as functions of time.

Fig. 10. Diffusion of pulsed magnetic field in theta-pinch geometry with $\mu_{1}=2$, $\mu_{2}=0.02, j_{c}=0.5$, and $\Delta j=0.01$. $\mathrm{B}_{\mathrm{zo}}=3$, supercritica1。

can extend over the entire plasma radius. This situation is not possible in the subcritical case $B_{z o}<r_{a} j_{c}$ shown in Fig. 11.
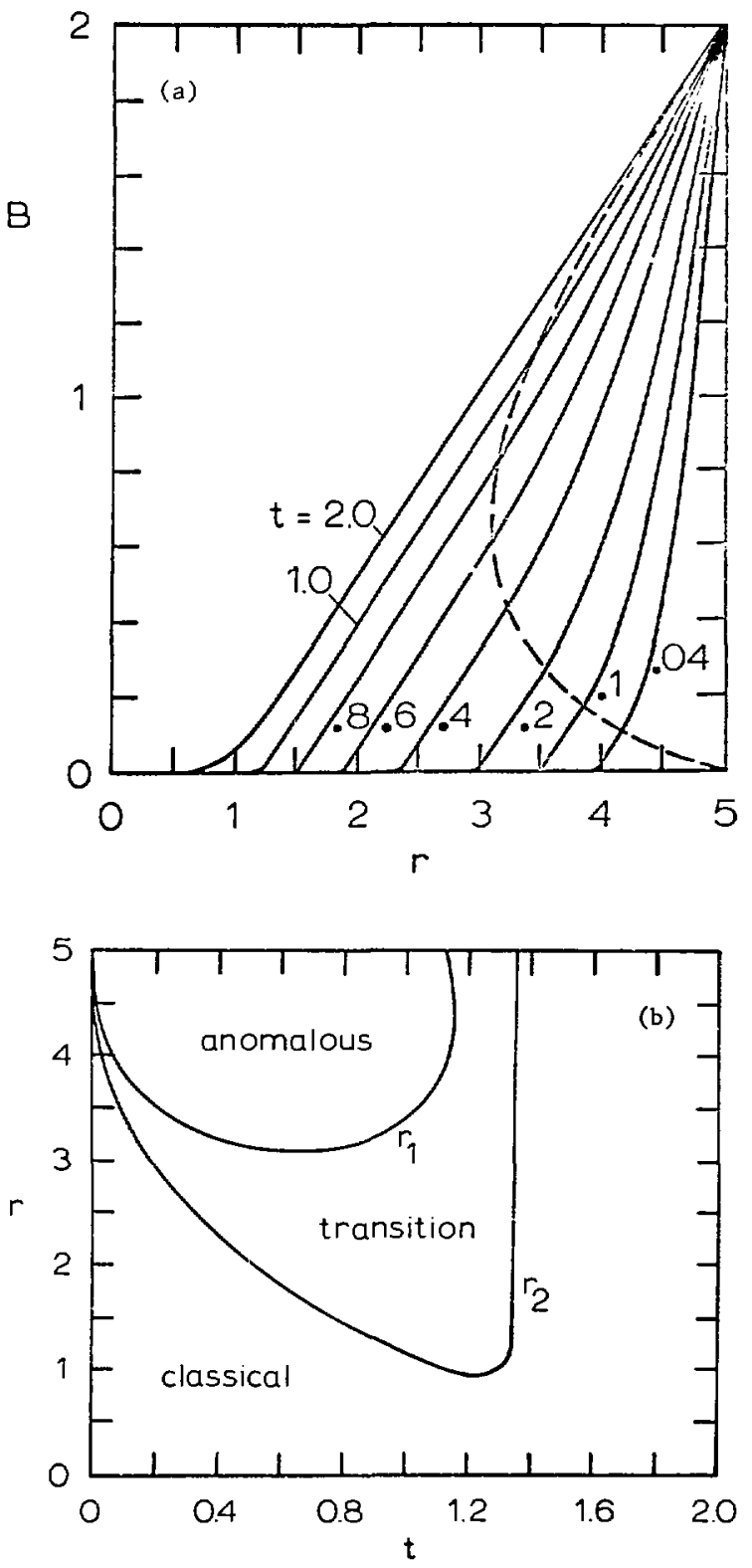

Fig. 11. Same as Fig. 10 with $B_{20}=2$, subcritical.

The speed of propagation of the transition region can be predicted in terms of its width $\left(r_{1}-r_{2}\right)$ and in its position. In the 1imit $\mu_{2} \rightarrow 0$,

$$
\frac{d r_{2}}{d t}=-\frac{2 r_{1} \mu_{1}}{r_{1}^{2}-r_{2}^{2}}
$$

This formula applies as long as the transition region does not reach the axis or the edge $r_{a}$ of the plasma column. 
The ${ }^{B}{ }_{\theta}$ field case is similar to a pure $Z$ pinch. $B_{\theta}$ falls as $1 / r$ in the outer region $r>r_{a}$, and the field at $x=x_{a}$ is pulsed from zero to $B_{e_{0}}$ at time $t=0$. The initial and boundary conditions are

$$
B= \begin{cases}0, & r<r_{a}, t<0, \\ B_{\theta 0}, & r=r_{a}, t \geq 0 .\end{cases}
$$

The diffusion equation becomes

$$
\frac{\partial B_{\theta}}{\partial t}=\frac{\partial}{\partial r}\left[\frac{\mu}{r} \frac{\partial}{\partial r}\left(\mathrm{rB}_{\theta}\right)\right] \text {, }
$$

where $\mu$ depends on $j=\left|j_{z}\right|$ and

$$
\mathrm{j}_{z}=\frac{1}{\mathrm{r}} \frac{\partial}{\partial \mathrm{r}}\left(\mathrm{rB}_{\theta}\right) \text {. }
$$

The explicit difference scheme is written in the form

$$
\begin{aligned}
B_{\theta, i+\frac{1}{2}}^{n+1}-B_{\theta, i+\frac{1}{2}}^{\mathrm{n}} & \\
= & \frac{\Delta t}{(\Delta \mathrm{r})^{2}}\left[\frac{\mu_{i+1}^{\mathrm{n}}}{r_{i+1}}\left(r_{i+3 / 2} B_{\theta, i+3 / 2}^{\mathrm{n}}-r_{i+\frac{1}{2}} B_{\theta, i+\frac{1}{2}}^{\mathrm{n}}\right)\right. \\
& \left.-\frac{\mu_{i}^{\mathrm{n}}}{r_{i}}\left(r_{i+\frac{1}{2}} B_{\theta, i+\frac{1}{2}}^{n}-r_{i-\frac{1}{2}} B_{\theta, i-\frac{1}{2}}^{n}\right)\right],
\end{aligned}
$$

where the $B_{\theta}$ flux is conserved everywhere except on the axis. The diffusion coefficient $\mu_{i}^{n}$ is defined in terms of Eq. (13) with

$$
j_{i}^{n}=\frac{1}{\Delta r} \frac{1}{r_{i}}\left(r_{i+\frac{1}{2}} B_{\theta, i+\frac{1}{2}}^{n}-r_{i-\frac{1}{2}} B_{\theta, i-\frac{1}{2}}^{n}\right) \text {. }
$$

Figures 12 and 13 show numerical solutions for $j_{c}=0.5, \Delta j=0.01, \mu_{1}=2$, end $\mu_{2}=0.02$. Superand subcritical are now defined as $B_{\theta 0}$ being 1 arger or smaller than $\mathbf{r}_{a} j_{c} / 2$, respectively. A supercritical $Z$ pinch eventually becomes entirely anomalous, whereas a subcritical pinch becomes completely classical.

The speed of propagation of the transition region, in the limit $\mu_{2} \rightarrow 0$, is given by

$$
\frac{\mathrm{dr}}{\mathrm{dt}}=-\frac{\mu_{1}}{\mathrm{r}_{2} \ln \left(\mathrm{r}_{1} / \mathrm{r}_{2}\right)}
$$

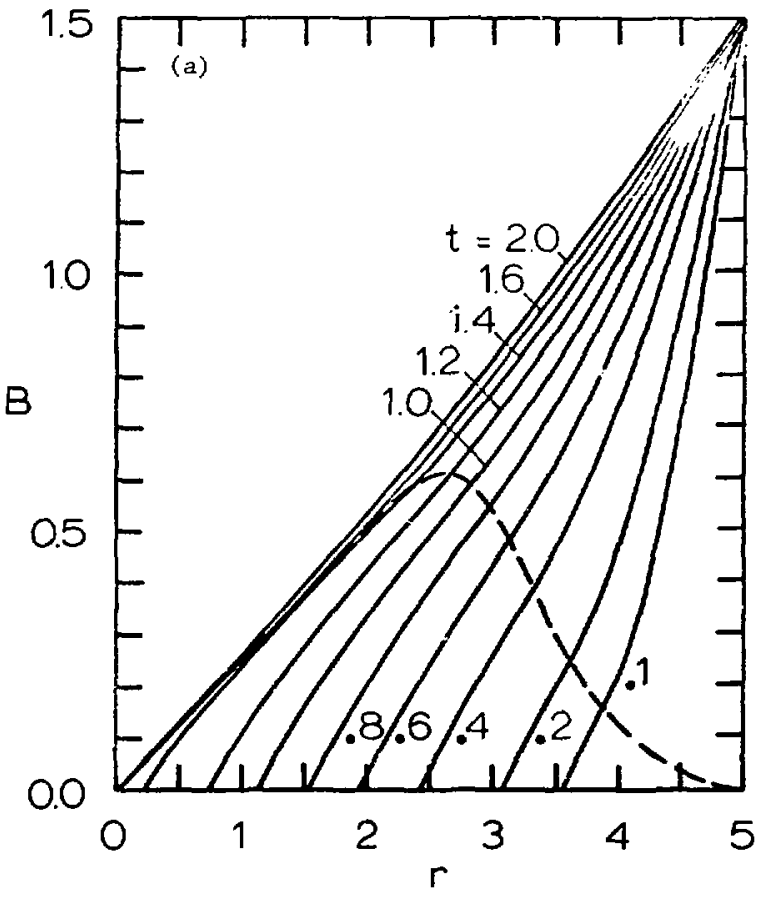

(a) $B_{\theta}(r)$ as a function of time. The dashed line represents $B_{\theta}\left(r_{1}\right) ; B_{\theta}\left(r_{2}\right)$ is close to the abscissa.

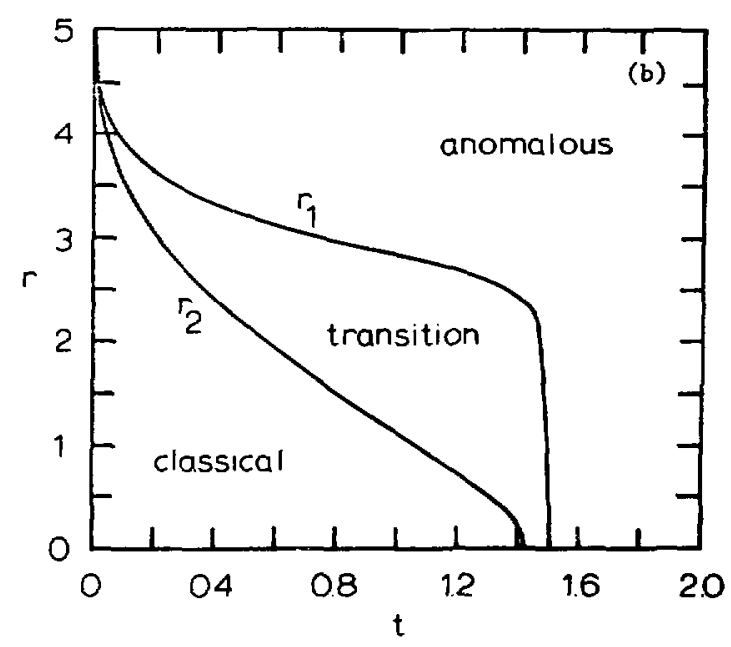

(b) $r_{1}$ and $r_{2}$ as functions of time.

Fig. 12. Diffusion of pulsed magnetic field in Zpinch geometry with $\mu_{1}=2, \mu_{2}=0.02$, $j_{c}=0.5$, and $\Delta j=0.01 . \quad B_{\theta 0}=1.5$, supercritical. 

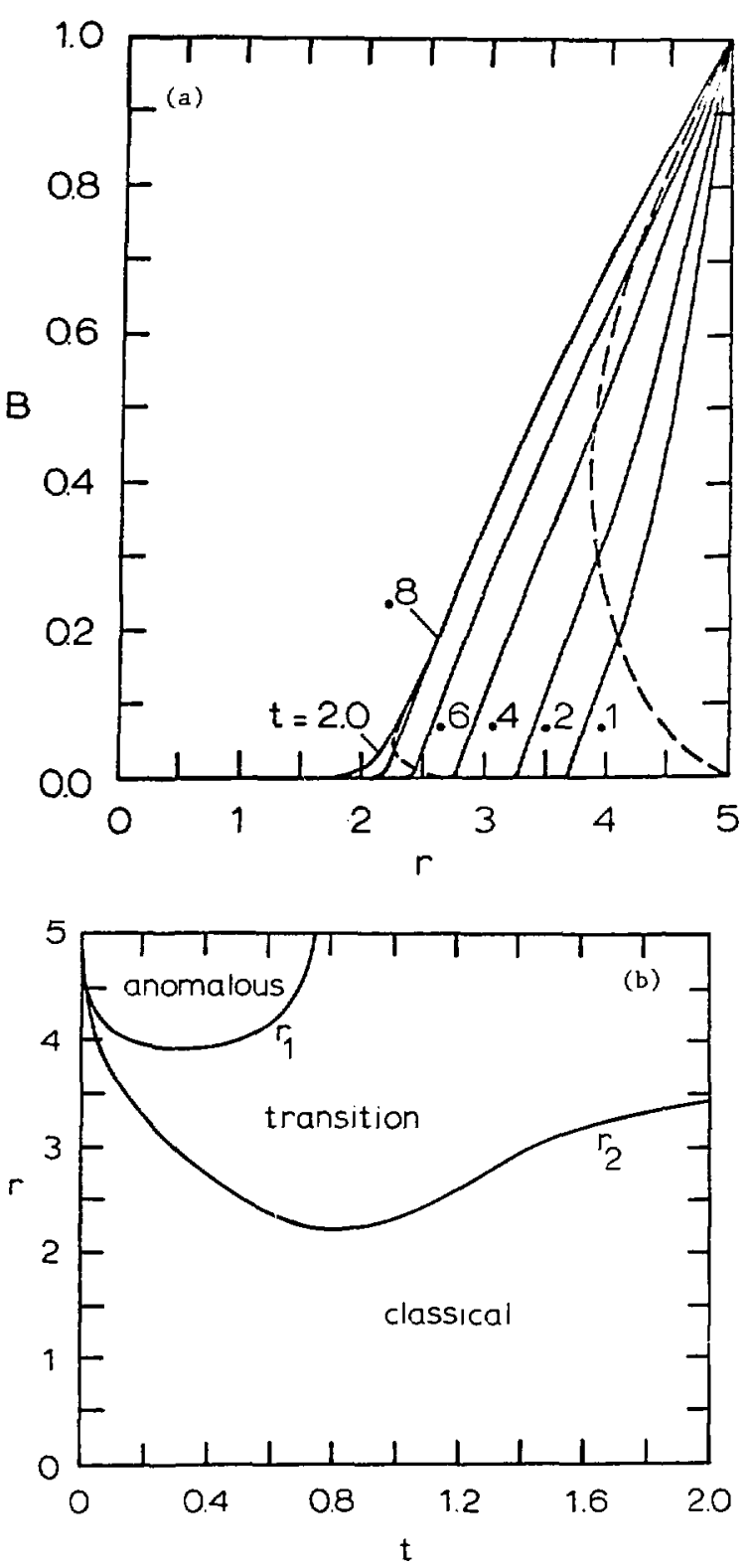

Fig. 13. Same as Fig. 12 with $B_{\theta 0}=1.0$, subcritical.

The results obtained in this section indicate that large regions of both the theta and $Z$ pinches can become part of the transition region. The current density can then be nearly uniform over a large portion of the discharges. This effect persists in the limit $\Delta j \rightarrow 0$. It is related to the fact that the magnetic flux must be conserved when $\mu_{2}$ changes to $\iota_{1}$.

\section{ACKNOWLEDGMENTS}

I wish to thank J. P. Freidberg, R. G. Kellner, M. M. Klein, J. A. Phillips, B. R. Suydam, B. K. Swartz, and $H$. Weitzner for their helpful discussions.

\section{REFERENCES}

1. H. A. B. Bodin, J. McCartan, A. A. Newton, and G. H. Wolf, "Diffusion and Stability of High- $\beta$ Plasma in an 8-Metre Theta Pinch," in Plasma Physics and Controlled Nuclear Fusion Research (International Atomic Energy Agency, Vienna, 1969), Vo1. 2, Pp. 533-553.

2. H. A. B. Bodin and A. A. Newton, "Study of the Diffusion of High- $\beta$ Plasma in a Theta Pinch," Phys. Fiuids 12, 2175-2184 (1969).

3. H. A. B. Bodin, J. McCartan, and G. H. Wolf, "The Early Stages of a Theta Pinch," in Third European Conference on Controlled Fusion and Plasma Physics (Wolters-Noordhoff Publishing, Groningen, 1969), p. 74.

4. A. Haberstich, "Status Report of the LASL Controlled Thermonuclear Research Program for 12Month Period Ending October 1970," Los Alamos Scientific Laboratory report LA-4585-MS (February 1971), PP. 37-39.

5. A. Haberstich, "Numerical Calculation of the Theta, 2, I Screw Pinches," Los Alamos Scientific laburatory report LA-4782-MS (December 19,1 ).

6. H. S, Carslew and J. C. Jaeger, Conduction of Heat in soljds (Oxford University Press, London, 1955), pp. 282-296.

7. R. D. Richtmyer, Difference Methods for InitialValue Problems (Interscience Publishers, Inc., New York, 1957), p. 104.

8. B. R. Suydam, Los Alamos Scientific Laboratory, personal communication, 1971.

9. R. D. Richtmyer, Difference Methods for InitialValue Problems (Interscience Publishers, Inc., New York, 1957), p. 93, Schenes 1 and 3 . 
APPENDIX A

STABILITY OF THE EXPLICIT SCHEME IN THE TRANSITION REGION

We expand both $B^{n}$ and $B^{n+1}$ to the third order in $: x$ about a mesh center point situated in the transition region and write

$$
\begin{aligned}
B_{i+1}^{n} & =a_{0} \\
B_{i-\vdots}^{n} & =a_{0}+a_{1} i x+a_{2}(x)^{2}, \\
B_{i+3 / 2}^{n} & =a_{0}-a_{1} \therefore x+a_{2}(A x)^{2},
\end{aligned}
$$

and

$$
\begin{aligned}
& B_{i+i}^{n+1}=b_{0} \\
& B_{i-i}^{n+1}=b_{0}+b_{1} A x+b_{2}(a x)^{2}, \\
& B_{i+3 / 2}^{n+1}=b_{0}-b_{1} i x+b_{2}(a x)^{2} .
\end{aligned}
$$

From Eqs. (13) and (14) and Eqs. (A-la) through $(A-1 c)$,

$$
a_{i}^{n}={ }_{i c}+\frac{i_{j}}{i j}\left(a_{1}-j_{c}+a_{2} a x\right)
$$

and

$$
{ }_{i 1}^{n}{ }_{i+1}=i_{c}+\frac{\dot{y}}{i j}\left(a_{1}-j_{c}-a_{2} \Delta x\right) .
$$

After substituting Eqs. $(A-1 a)$ through $(A-3 b)$ into the explicit difference equation, Eq. (11), we find that

$b_{0}-a_{0}=2 k t a_{2}\left[\mu_{c}+\frac{\Delta \mu}{\Delta j}\left(2 a_{1}-j_{c}\right)\right]$

To determine the stability of the explicit scheme, we add a small perturbation of wavelength $2 i x$ and amplitude $\epsilon_{a}$ to $B^{n}$.
Thus,

$$
\begin{aligned}
& B_{i+:}^{n}=a_{0}+a_{a} \\
& B_{i-:}^{n}=a_{0}+a_{1} \cdot x+a_{2}(\vdots x)^{2}-a_{a}, \\
& \text { and } \\
& B_{i+3 / 2}^{n}=a_{0}-a_{1} i x+a_{2}(x x)^{2}-a_{a} \text {. }
\end{aligned}
$$

Then

$$
{ }_{i}^{n}=u_{c}+\frac{j}{\vdots j}\left[a_{1}-j_{c}+\left(a_{2} \cdot d x-2 \frac{\dot{z}_{a}}{\vdots x}\right)\right]
$$

and

$$
{ }_{i+1}^{n}=\therefore c+\frac{1}{4 j}\left[a_{1}-j_{c}-\left(a_{2} a x-2 \frac{i}{i x}\right)\right] \text {. }
$$

We assume that this perturbation gives rise to a similar perturbation $\varepsilon_{b}$ in $B^{\text {nfl }}$, such that

$$
\begin{aligned}
& B_{i+:}^{n+1}=b_{0}+b_{b} \\
& B_{i-i}^{n+1}=b_{0}+b_{1} A x+b_{2}(j x)^{2}-b_{b},
\end{aligned}
$$

and

$B_{i+3 / 2}^{n+1}=b_{0}-b_{1} 1 x+b_{2}(n x)^{2}-a_{b}$ $(A-7 c)$

Substituting Eqs. (A-5a) through $(A-7 c)$ into the difference equation, Eq. (11), we obtain

$$
\begin{aligned}
& \left(b_{0}-a_{o}\right)+\left(z_{b}-a_{a}\right)= \\
& 2 a t\left[a_{2}-2 \frac{z_{a}}{(s: x)^{2}}\right]\left[1 c_{c}+\frac{1}{1 j}\left(2 a_{1}-j_{c}\right)\right]
\end{aligned}
$$


We now subtract the unperturbed $\mathrm{Eq}$. (A-4) trom Eq. $(A-8)$, solve for $i b$, and $f$ ind

$$
\bar{c}_{b}=\gamma \ddot{i 1}_{1} \quad,
$$

where

$$
\gamma=1-4 \frac{i t}{(x x)^{2}}\left[c+\frac{i}{i}\left(2 a_{1}-i_{c}\right)\right]
$$

For stability, $y$ must satisty $|\gamma|=1$, that is,

$$
4 \frac{i t}{(i x)^{2}}\left[\ddots_{c}+\frac{i}{i j}\left(2 a_{1}-i_{c}\right)\right] \div 2 \cdot(A-11)
$$

The worst condition occurs for $a_{1}=i_{c}+-1 / 2$. The stability condition then becomes

$$
\frac{i t}{(j x)^{2}} c-\frac{1}{1+\frac{i c}{c}\left(1+\frac{i c}{i j}\right)}
$$

Equation $(A-12)$ is plotted in Fig. $A-1$ where it is compared against stability limits abserved experimentally on the computer.

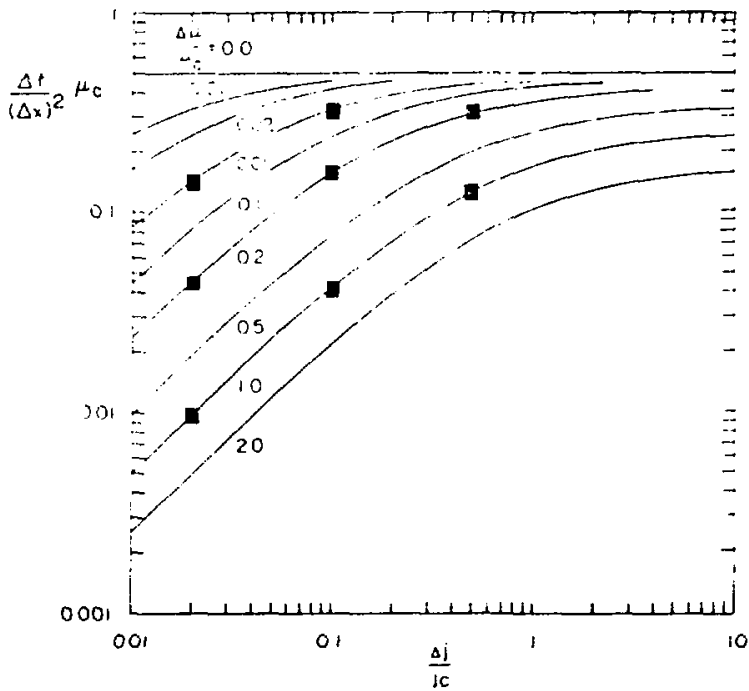

Fig. A-1. Explicit stability limits of,$a t /(\therefore x)^{2}$ as functions of $\therefore \mathrm{j} / \mathrm{i}_{\mathrm{c}}$ for several values of ... /. The curves are calculated from Eq. $(A-12)$. The squares are limits observed with $x_{2}-x_{1}=2.04$ and $\mathrm{x} x=$ 0.05 .

APPENDIX B

STABILITY OF THE IMPLICIT SCHEME IN THE TRANSITION REGION

We assume that the implicit difference equation, Eq. (12), has been solved exactly. $B^{n+l}$ is then given by Eqs. $(A-2 a)$ chrough $(A-2 C)$,

$$
\cdot_{i}^{n+1}=n_{c}+\frac{y_{i}}{a j}\left(b_{1}-i_{c}+b_{2}(x),\right.
$$

and

$$
{ }_{n+1}^{n+1}=n_{c}+\frac{\vdots j}{\vdots j}\left(b_{1}-j_{c}-b_{2} \cdot x\right) \cdot(B-1 b) \text { and }
$$

Substituting Eqs. ( $A-1 a)$ through $(A-2 C)$ and Eqs. $(B-1 a)$ and $(B-1 b)$ into the difference equation, $E q$. (12), we find that

$$
b_{0}-a_{0}=2: t b_{2}\left[c_{c}+\frac{\partial j}{\therefore j}\left(2 b_{1}-j_{c}\right)\right] \cdot(B-2)
$$

To determine the stability of the iteration scheme, Eq. (i7), we add a small perturbation of wavelength $2 i x$ and amplitude $\dot{ }_{;}$, to the diffusion coefficient. Then

$$
u_{i}^{n+l, m}=n_{c}+\frac{\hat{u}^{1}}{1 j}\left(b_{1}-j_{c}+b_{2} i x\right)+\sum_{i}^{m}
$$

$(B-3 a)$

and

$$
{ }_{i+1}^{n+1, m}=a_{c}+\frac{1 \mu}{\therefore j}\left(b_{1}-i_{c}-b_{2} i x\right)-{ }_{n}^{m i} .
$$

We assume that this perturbation causes a similar perturbation of amplitude $\dot{z}_{b}$ in $B^{n+1}$. Substituting Eqs. $(A-1 a)$ through $(A-1 C)$, Eqs. $(A-7 a)$ through 
$(A-7 c)$, as well as Eqs. $(B-3 a)$ and $(B-3 b)$ into the implicit difference equation, Eq. (12), we find that

$$
\begin{aligned}
& b_{0}-a_{0}+\varepsilon_{b}^{m}=2 \Delta t\left\{b_{2}\left[\mu_{c}+\frac{\Lambda \mu}{\Delta j}\left(2 b_{1}-j_{c}\right)\right]\right. \\
& \left.+b_{1} \frac{\varepsilon_{\mu}^{m}}{\wedge x}-2 \frac{\varepsilon_{b}^{m}}{(\Lambda x)^{2}}\left[\mu_{c}+\frac{\Lambda \mu}{\Delta j}\left(b_{1}-j_{c}\right)\right]\right\} .
\end{aligned}
$$

We subtract the unperturbed $\mathrm{Eq} .(\mathrm{B}-2)$ from Eq. $(B-4)$, solve for $\varepsilon_{b}^{m}$, and obtain

$$
\epsilon_{b}^{m}=\gamma \epsilon_{\mu}^{m}
$$

where

$$
\gamma=2 \frac{\Delta t}{(\Delta x)^{2}} \frac{b_{1} \Delta x}{1+4 \frac{\Delta t}{(\Delta x)^{2}\left[\mu_{c}+\frac{\Delta \mu}{\Delta j}\left(b_{1}-j_{c}\right)\right]}} .
$$

In terms of $B^{n+1, m}$,

$$
\bar{\mu}_{i}^{n+1, m}=\mu_{c}+\frac{\Delta \mu}{\Delta j}\left[b_{1}-j_{c}+\left(b_{2} \Delta x-2 \frac{\epsilon_{b}^{m}}{\Delta x}\right)\right]
$$

and

$$
\bar{\mu}_{i+1}^{n+1, m}=\mu_{c}+\frac{\Delta \mu}{\Delta j}\left[b_{1}-j_{c}-\left(b_{2} \Delta x-2 \frac{\varepsilon_{b}^{m}}{\Delta x}\right)\right] .
$$

With these coefficients and using Eq. (17), $\mu^{\mathrm{n}+1, \mathrm{~m}+1}$ becomes

$$
\mu_{i}^{n+1, m+1}=\mu_{c}+\frac{\Delta \mu}{\Delta j}\left(b_{1}-j_{c}+b_{2} \Delta x\right)+\varepsilon_{\mu}^{m+1},
$$

where

$$
\epsilon_{\mu}^{m+1}=\epsilon_{\mu}^{m}\left[(1-A)-2 A \frac{\Delta_{\mu}}{\Delta j} \frac{\gamma}{\Delta x}\right] .
$$

For stability, it is necessary that

$$
\left|\varepsilon_{\mu}^{m+1}\right| \leq\left|\varepsilon_{\mu}^{m}\right|
$$

that is,

$$
A\left(1+2 \frac{\Delta_{\mu}}{\Delta j} \frac{\gamma}{\Delta x}\right) \leq 2
$$

Substituting for $\gamma$, we obtain the stability condition

$$
4 \frac{\Delta t}{(\Delta x)^{2}} \leq \frac{\frac{2}{A}-1}{b_{1} \frac{\Delta L}{\Delta j}-\left(\frac{2}{A}-1\right)\left[\mu_{c}+\frac{\Delta L}{\Delta j}\left(b_{1}-j_{c}\right)\right]}
$$

The right-hand side of this expression is smallest for $b_{1}=j_{c}-1 j / 2$. The stabiliry condition then becomes

$\frac{\Delta t}{(\Delta x)^{2}} \mu_{c} \leq \frac{\frac{2}{A}-1}{\frac{\Delta \mu}{\mu_{c}}\left[\left(\frac{1}{A}-1\right)+\frac{j_{c}}{\Delta j}\right]-\left(\frac{2}{A}-1\right)}$.

Equation (B-13) is plotted in Fig. B-1 for $\Delta \psi / \mu_{c}=1 / 1.5$. It is compared against stability limits observed experimentally on the computer.

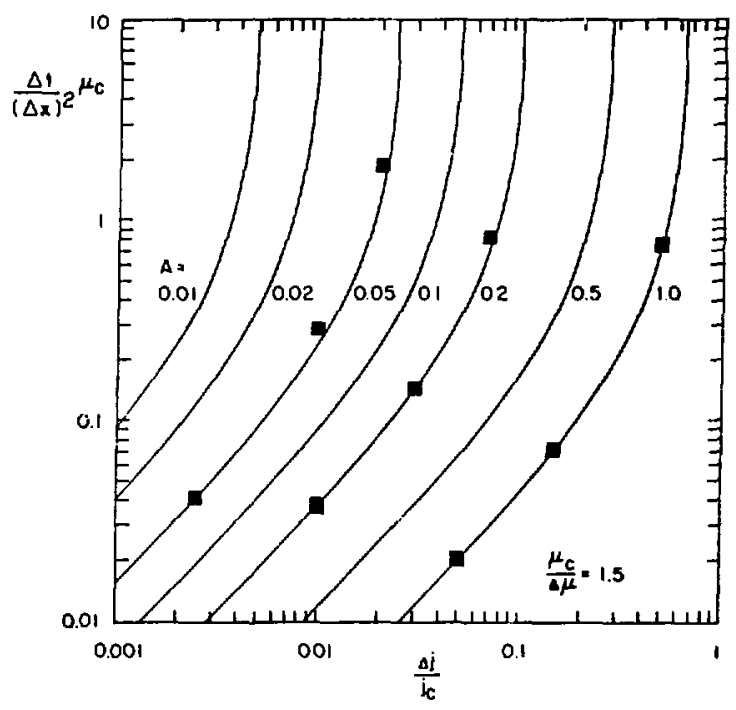

Fig. B-1. Implicit limits of $\mu_{c} \Delta t /(\Delta x)^{2}$ as functions of $A j / j$ for several values of the relaxation factor $A$, with $\Delta \mu / \mu_{c}=1 / 1.5$. The curves are calculated from Eq. (B-13). The squares are limits observed with $\mu_{1}=2, \mu_{2}=1$, and $\Delta x=0.05$.

A. U. S. GOYERNMENT PRINTING OFFICE: 1973 - 784-278/20 